\title{
On Rotations as Spin Matrix Polynomials
}

\author{
T. L. Curtright ${ }^{\S}$ and T. S. Van Kortryk \\ curtright@miami.edu and vankortryk@gmail.com \\ $\S$ Department of Physics, University of Miami \\ Coral Gables, FL 33124-8046, USA
}

\begin{abstract}
Recent results for rotations expressed as polynomials of spin matrices are derived here by elementary differential equation methods. Structural features of the results are then examined in the framework of biorthogonal systems, to obtain an alternate derivation. The central factorial numbers play key roles in both derivations.
\end{abstract}

\section{Introduction}

Curtright, Fairlie, and Zachos (CFZ) recently obtained explicit and intuitive results 1 expressing the rotation matrix for any quantized angular momentum $j$ as a polynomial of order $2 j$ in the corresponding $(2 j+1) \times(2 j+1)$ spin matrices $\hat{\boldsymbol{n}} \cdot \boldsymbol{J}$ that generate rotations about axis $\hat{\boldsymbol{n}}$. While many previous studies of this or closely related problems can be found in the literature - beginning with the work of Wigner in the 1930s 2, 3] and then, after a lengthly hiatus, continuing in the 1960s and subsequently with direct attacks on the problem by Lehrer-Ilamed [4], van Wageningen [5], and others [6]- [] - none of these other studies succeeded to find such simple, compact expressions for the coefficients in the spin matrix polynomial, as elementary functions of the rotation angle, as those obtained by CFZ. For each angle-dependent coefficient in the polynomial, the explicit formula found by CFZ involves nothing more complicated than a truncated series expansion for a power of the arcsin function.

The CFZ formula for a rotation through an angle $\theta$ about an axis $\hat{\boldsymbol{n}}$, valid for any spin $j \in\left\{0, \frac{1}{2}, 1, \frac{3}{2}, \cdots\right\}$, is

$$
\exp (i \theta \hat{\boldsymbol{n}} \cdot \boldsymbol{J})=\sum_{k=0}^{2 j} \frac{1}{k !} A_{k}^{[j]}(\theta)(2 i \hat{\boldsymbol{n}} \cdot \boldsymbol{J})^{k}
$$

where the angle-dependent coefficients of the various spin matrix powers are given simply by

$$
A_{k}^{[j]}(\theta)=\sin ^{k}(\theta / 2) \quad(\cos (\theta / 2))^{\epsilon(j, k)} \operatorname{Trunc}_{\lfloor j-k / 2\rfloor}\left[\frac{1}{(\sqrt{1-x})^{\epsilon(j, k)}}\left(\frac{\arcsin \sqrt{x}}{\sqrt{x}}\right)^{k}\right]_{x=\sin ^{2}(\theta / 2)} .
$$

Here, $\lfloor\cdots\rfloor$ is the integer-valued floor function 11 and $\operatorname{Trunc}_{n}[f(x)]$ is the $n$ th-order Taylor polynomial truncation for any $f(x)$ admitting a power series representation:

$$
f(x)=\sum_{m=0}^{\infty} f_{m} x^{m}, \quad \underset{n}{\operatorname{Trunc}}[f(x)] \equiv \sum_{m=0}^{n} f_{m} x^{m} .
$$

In addition, $\epsilon(j, k)$ is a binary-valued function 2 of $2 j-k$ that distinguishes even and odd integers: $\epsilon(j, k)=0$ for even $2 j-k$, and $\epsilon(j, k)=1$ for odd $2 j-k$.

As observed in [1, the results (2) display the limit $j \rightarrow \infty$ for fixed $k$ in a beautifully intuitive way. In that limit, the truncation is lifted to obtain trigonometrical series for the periodicized $\theta^{k}$ monomials. But even as $j \rightarrow \infty$, integer $j$ (bosonic) and semi-integer $j$ (fermionic) coefficients are clearly distinguished by a relative sign flip for $\theta \in[\pi, 3 \pi] \bmod (4 \pi)$. This is evident upon plotting the first few coefficients for very large spins. Following [1], a few examples are shown in Appendix G.

\footnotetext{
${ }^{1}$ For any $r \in \mathbb{R},\lfloor r\rfloor=\sup \{n \in \mathbb{Z}$ such that $n \leq r\}$.

${ }^{2}$ For $k$ integer and $j$ either integer or semi-integer, $\epsilon(j, k)=\left(1-(-1)^{2 j-k}\right) / 2$.
} 
In practice, for finite $j$ of reasonable size, the truncations needed to evaluate (2) are easily obtained as a matter of course by machine computation, for example by using either Maple or Mathematica. Nevertheless, it is interesting and relevant for the analysis to follow that Taylor series for powers of cyclometric functions can be expressed in terms of $t(m, n)$, the so-called central factorial numbers of the first kind [14, 15. Thus for $|z| \leq 1$ and non-negative integer $n$ (cf. Theorem (4.1.2) in [15]),

$$
(\arcsin (z))^{n}=\frac{n !}{2^{n}} \sum_{m=n}^{\infty} \frac{|t(m, n)|}{m !}(2 z)^{m} .
$$

Note that the coefficients in these Taylor series are all non-negative. Also, $t(m, n)=0$ for odd $m+n$, so the expansions (4) for even (odd) powers of $\arcsin (z)$ are indeed even (odd) functions of $z$. In general, the values of $t(m, n)$ are defined by and obtained from simple polynomials, as described in Appendix A.

Incorporating (4) into the expression for the coefficients (2) gives

$$
A_{k}^{[j]}(\theta)=\frac{k !}{2^{k}} \sum_{m=k}^{2 j} \frac{2^{m}}{m !}|t(m, k)| \sin ^{m}(\theta / 2) \quad \text { for even } 2 j-k .
$$

As firmly established in [5, 1], the remaining coefficients in (11) may then be obtained from

$$
A_{k-1}^{[j]}(\theta)=\frac{2}{k} \frac{d}{d \theta} A_{k}^{[j]}(\theta) \quad \text { for odd } 2 j-k+1 .
$$

\section{A Derivation Using Differential Equations}

The goal here is to derive (5), hence to establish (2) and (1), by using elementary results extant in the literature, and by using a simple Lemma, namely,

$$
(2 \hat{\boldsymbol{n}} \cdot \boldsymbol{J})^{2 j+1}=-\sum_{m=0}^{2 j} 2^{1+2 j-m} \times t(2+2 j, 1+m) \times(2 \hat{\boldsymbol{n}} \cdot \boldsymbol{J})^{m} .
$$

This Lemma is established in Appendix B. Given the Lemma and well-known properties of the central factorial numbers, a proof of (5) follows directly. To see this, begin by considering integer values of $j$.

For integer $j$ and even index coefficients, the results

$$
A_{2 k}^{[j]}(\theta)=\frac{(2 k) !}{4^{k}} \sum_{m=2 k}^{2 j} \frac{2^{m}}{m !}|t(m, 2 k)| \sin ^{m}(\theta / 2)
$$

may be obtained by verifying that these series for various $k$ are in fact solutions of the second-order equations

$$
A_{2 k-2}^{[j]}(\theta)=\frac{4}{2 k(2 k-1)} \frac{d^{2}}{d \theta^{2}} A_{2 k}^{[j]}(\theta)+(-4)^{j-k+1} \times t(2+2 j, 2 k) \times \frac{(2 k-2) !}{(2 j) !} A_{2 j}^{[j]}(\theta),
$$

with proper behavior near $\theta=0$. The correct small $\theta$ behavior follows immediately from that of the exponential on the LHS of (1), and is easily seen to hold for the series (8) and their first derivatives with respect to $\theta$. That these second-order differential equations and initial conditions are necessary and sufficient is a straightforward consequence of the first derivative relations carefully derived in Section 6 of [1, and of the Lemma. By dealing with the second-order equation (9) instead of directly with first derivatives of the coefficients, one can avoid the cosine and $\sqrt{1-x}$ factors in (2). The coefficients must also obey higher order differential equations, as discussed in [5, 7. These higher order equations are also satisfied by (8), but it is unnecessary to show this here.

To show that (9) is indeed satisfied by (8), first compute the second derivative of the series:

$$
\frac{4}{2 k(2 k-1)} \frac{d^{2}}{d \theta^{2}} A_{2 k}^{[j]}(\theta)=\frac{(2 k-2) !}{4^{k-1}} \sum_{m=2 k}^{2 j}\left(\frac{2^{m-2}}{(m-2) !}|t(m, 2 k)| \sin ^{m-2}(\theta / 2)-\frac{2^{m}}{m !} \frac{m^{2}}{4}|t(m, 2 k)| \sin ^{m}(\theta / 2)\right) .
$$

Then compare this to (8) for $2 k \rightarrow 2 k-2$, after rewriting the latter by making use of the elementary recurrence formulas for the central factorial numbers, as given in Proposition 2.1 of [15], say. In particular, $t(m, n)=0$ for $m<n$, and otherwise

$$
t(m, 2 k-2)=t(m+2,2 k)+\frac{1}{4} m^{2} t(m, 2 k) .
$$


Using this recurrence relation in (8) gives

$$
\begin{aligned}
A_{2 k-2}^{[j]}(\theta) & =\frac{(2 k-2) !}{(2 j) !} \frac{4^{j}}{4^{k-1}}|t(2+2 j, 2 k)| \sin ^{2 j}(\theta / 2)+\frac{(2 k-2) !}{4^{k-1}} \sum_{m=2 k}^{2 j} \frac{2^{m-2}}{(m-2) !}|t(m, 2 k)| \sin ^{m-2}(\theta / 2) \\
& -\frac{(2 k-2) !}{4^{k-1}} \sum_{m=2 k}^{2 j} \frac{2^{m}}{m !} \frac{m^{2}}{4}|t(m, 2 k)| \sin ^{m}(\theta / 2),
\end{aligned}
$$

upon assigning the correct phases (see (A2) in Appendix A). But the highest coefficient $A_{2 j}^{[j]}$ is readily shown to be (e.g. see [1])

$$
A_{2 j}^{[j]}(\theta)=\sin ^{2 j}(\theta / 2) .
$$

Therefore (9) and (8) are verified.

For semi-integer $j$ a similar derivation involving the odd index coefficients $A_{2 k+1}^{[j]}(\theta)$ goes through perfectly in parallel to the even index case, and thereby completes this derivation of the CFZ results.

\section{A Derivation Using Biorthogonality}

Considerable analysis and combinatorics are implicit in (11) and (2). Perhaps the analytic features of the CFZ formulas are most fully appreciated if viewed in the context of biorthogonal systems. In any case, the theory of biorthogonal systems naturally leads to another proof of (1).

\section{Biorthogonal Functions}

Since the Taylor polynomials produced by the truncation in (2) involve only even powers of $\sin (\theta / 2)$ with nonnegative coefficients, the resulting set of polynomials are not orthogonal for any positive measure on $\theta$. Instead, the dual function space consists of linear combinations of Chebyshev polynomials $(\cos (k \theta)$ with $0 \leq k \leq j$, for any fixed integer value of $j$ ) and these linear combinations alternate in sign as functions of $\theta$ to give the requisite orthogonality. This provides, in a quantum physics context, an elementary example of a finitely indexed biorthogonal system of functions [10]. (For examples of infinite and countably indexed biorthogonal quantum systems, see [11, 12. For other examples and a careful discussion of the relevant theory, see [13].)

To understand this structure, consider a basis of monomials of even powers of $\sin (\theta / 2)$. The $j+1$ lowest powers of $\sin ^{2}(\theta / 2)$, beginning with $1=\sin ^{0}(\theta / 2)$ and ending with $\sin ^{2 j}(\theta / 2)$, constitute one half of a finite biorthogonal system of functions $f$ and their duals $g:\left\{f_{n}^{[j]}, g_{n}^{[j]} \mid n=0,1, \cdots, j\right\}$. For integer $j$ the functions and their duals are given by

$$
\begin{aligned}
& f_{0}^{[j]}(\theta)=1 \quad \text { and } \quad g_{0}^{[j]}(\theta)=1+2 \sum_{k=1}^{j} \cos (k \theta) \quad \text { for } n=0, \\
& f_{n}^{[j]}(\theta)=\sin ^{2 n}(\theta / 2) \quad \text { and } \quad g_{n}^{[j]}(\theta)=(-4)^{n} \sum_{k=n}^{j} \frac{k}{n}\left(\begin{array}{c}
k+n-1 \\
2 n-1
\end{array}\right) \cos (k \theta) \quad \text { for } 0<n \leq j .
\end{aligned}
$$

These are orthogonal and normalized for any particular $j$ :

$$
\delta_{m, n}=\frac{1}{2 \pi} \int_{-\pi}^{+\pi} g_{m}^{[j]}(\theta) f_{n}^{[j]}(\theta) d \theta
$$

In the spirit of (2) the dual functions may be written as truncations of infinite series in the variable $w=e^{i \theta}$, namely,

$$
g_{n}^{[j]}(\theta)=\operatorname{Re}\left(h_{n}^{[j]}(\theta)\right) \quad \text { where } \quad h_{n}^{[j]}(\theta)=\operatorname{Trunc}_{j}\left[\frac{(-4 w)^{n}(1+w)}{(1-w)^{1+2 n}}\right]_{w=e^{i \theta}} \quad \text { for } 0<n \leq j .
$$

There are similar results for a finite biorthogonal system of functions consisting of the odd powers $\sin ^{2 n-1}(\theta / 2)$, and their duals. This other system is easily obtained from the biorthogonal system involving the even powers of $\sin (\theta / 2)$, as given above, just by moving a single factor of $\sin (\theta / 2)$ from the functions to the dual functions. For 
application to the spin matrix expansion, consider semi-integer $j$. The $j+1 / 2$ lowest odd powers of $\sin (\theta / 2)$, beginning with $\sin (\theta / 2)$ and ending with $\sin ^{2 j}(\theta / 2)$, again constitute one half of a biorthogonal system of functions $f$ and their duals $g:\left\{f_{n}^{[j]}, g_{n}^{[j]} \mid n=1, \cdots, j+1 / 2\right\}$. For semi-integer $j$ the functions and their duals are now given by

$$
f_{n}^{[j]}(\theta)=\sin ^{2 n-1}(\theta / 2) \quad \text { and } \quad g_{n}^{[j]}(\theta)=(-4)^{n} \sin (\theta / 2) \sum_{k=n}^{j+1 / 2} \frac{k}{n}\left(\begin{array}{c}
k+n-1 \\
2 n-1
\end{array}\right) \cos (k \theta) \quad \text { for } 1 \leq n \leq j+1 / 2 .
$$

Once again, these are orthogonal and normalized as in (15). Note that the dual functions of the latter system are orthogonal to all even powers of $\sin (\theta / 2)$. Equivalently, the dual functions of the biorthogonal system discussed previously are orthogonal to all odd powers of $\sin (\theta / 2)$. Thus the two systems may be combined into a larger one, involving both even and odd powers of $\sin (\theta / 2)$, without modification of the dual functions. To be even more explicit, additional details are given in Appendix D, including some useful Tables.

\section{Biorthogonal Matrices}

Next, consider dual matrices which are trace orthonormalized with respect to powers of the spin matrix, $S \equiv$ $2 \hat{\boldsymbol{n}} \cdot \boldsymbol{J}$. Without loss of generality, choose $S=2 J_{3}$, since any other choice for $\hat{\boldsymbol{n}}$ merely requires selecting a different basis to diagonalize the spin matrix, thereby obtaining the same eigenvalues as $2 J_{3}$. Thus the powers are

$$
S^{m}=\left(\begin{array}{ccccc}
(2 j)^{m} & 0 & \cdots & 0 & 0 \\
0 & (2 j-2)^{m} & \cdots & 0 & 0 \\
\vdots & \vdots & \ddots & \vdots & \vdots \\
0 & 0 & \cdots & (-2 j+2)^{m} & 0 \\
0 & 0 & \cdots & 0 & (-2 j)^{m}
\end{array}\right)
$$

Now construct orthonormalized dual matrices $T_{n}$ such that

$$
\delta_{n, m}=\operatorname{Trace}\left(T_{n} S^{m}\right), \quad n, m=0,1, \cdots, 2 j .
$$

Clearly, the $T_{n}$ may also be chosen to be diagonal $(2 j+1) \times(2 j+1)$ matrices in the basis that diagonalizes $S$. In fact, for any spin $j$ the required entries on the diagonal of $T_{n}$ are just the entries in the $(n+1)$ st row of the inverted Vandermonde matrix, $V^{-1}[j]$. (Note that here, unlike the conventions in [1, both rows and columns of the Vandermonde matrix and its inverse are indexed as $1,2, \cdots, 2 j+1$.) That is,

$$
\left(T_{n-1}\right)_{k k}=\left(V^{-1}[j]\right)_{n, k}, \quad n, k=1, \cdots, 2 j+1 .
$$

This result follows immediately from the fact that the diagonal entries for $S^{m}$ are just the entries in the corresponding column (i.e. the $(m+1)$ st column) of the Vandermonde matrix, $V[j]$.

$$
V[j]=\left(\begin{array}{ccccc}
1 & 2 j & (2 j)^{2} & \cdots & (2 j)^{2 j} \\
1 & 2 j-2 & (2 j-2)^{2} & \cdots & (2 j-2)^{2 j} \\
\vdots & \vdots & \vdots & \ddots & \vdots \\
1 & -2 j & (-2 j)^{2} & \cdots & (-2 j)^{2 j}
\end{array}\right) .
$$

Thus the effective metric, $G$, on the space spanned by powers of the spin $j$ matrices, defined such that

$$
\delta_{m, n}=S^{m} \cdot G \cdot S^{n} \equiv \sum_{k, l}\left(S^{m}\right)_{k k} G_{k l}\left(S^{n}\right)_{l l},
$$

is given by $G=\left(V^{-1}\right)^{\dagger}\left(V^{-1}\right)$. That is to say, since $V$ and $V^{-1}$ are real in the chosen basis, the metric is

$$
G_{k l}[j]=\sum_{i} V^{-1}[j]_{i k} V^{-1}[j]_{i l} .
$$

Another way to write the orthonormality (22) is by incorporating the metric $G$ into a matrix trace.

$$
\delta_{m, n}=\operatorname{Trace}\left(B S^{m} G S^{n}\right),
$$

where $B$ is a singular matrix with all entries equal to 1 .

We have more to say about (24) in Appendix F, but first we encourage the reader to consider the explicit examples of spin matrix powers, their duals, the corresponding Vandermonde matrix and its inverse $V^{-1}[j]$, and the metric $G$, for $j=1 / 2,1,3 / 2$, and 2 , as given in Appendix E. 


\section{Extracting the Coefficients}

Returning to the problem at hand, for any given spin $j$, the dual matrices may be used to extract the individual angle-dependent coefficients in the expansion of the rotation matrix, (1), in the basis that diagonalizes $\hat{\boldsymbol{n}} \cdot \boldsymbol{J}$. That is,

$$
A_{k}^{[j]}(\theta)=(-i)^{k} k ! \text { Trace }\left[T_{k} e^{i \theta(\hat{\boldsymbol{n}} \cdot \boldsymbol{J})}\right]=(-i)^{k} k ! \operatorname{Trace}\left[T_{k}\left(\begin{array}{cccc}
e^{i j \theta} & 0 & \cdots & 0 \\
0 & e^{i(j-1) \theta} & \cdots & 0 \\
\vdots & \vdots & \ddots & \vdots \\
0 & 0 & \cdots & e^{-i j \theta}
\end{array}\right)\right] .
$$

Consider for now only integer $j$ and even $k \in\{0, \cdots, 2 j\}$. From (25) the form

$$
\left.A_{k}^{[j]}(\theta)\right|_{\substack{j \text { integer } \\ k \text { even }}}=\sum_{n=k / 2}^{j} a_{k, n}^{[j]} \sin ^{2 n}(\theta / 2)
$$

may be argued to hold from generic behavior of the coefficients under Fourier analysis (periodicity in $\theta$, symmetry under reflections, etc.). More specifically, for integer $j$ and even $k \in\{0, \cdots, 2 j\}$, with $n \in\{k / 2, \cdots, j\}$, the previous dual functions for even powers of $\sin (\theta / 2)$ may be used to extract the coefficients $a_{k, n}^{[j]}$ as

$$
\begin{aligned}
a_{k, n}^{[j]} & =\frac{1}{2 \pi} \int_{-\pi}^{+\pi} g_{n}^{[j]}(\theta) A_{k}^{[j]}(\theta) d \theta \\
& =\frac{(-i)^{k} k !}{2 \pi} \operatorname{Trace}\left[T_{k}\left(\begin{array}{cccc}
\int_{-\pi}^{+\pi} g_{n}^{[j]}(\theta) e^{i j \theta} d \theta & 0 & \cdots & 0 \\
0 & \int_{-\pi}^{+\pi} g_{n}^{[j]}(\theta) e^{i(j-1) \theta} d \theta & \cdots & 0 \\
\vdots & \vdots & \ddots & \vdots \\
0 & 0 & \cdots & \int_{-\pi}^{+\pi} g_{n}^{[j]}(\theta) e^{-i j \theta} d \theta
\end{array}\right)\right] .
\end{aligned}
$$

As established in (201), the diagonal elements $\left(T_{k}\right)_{n, n}$ are just the entries in the $n$th column of the $(k+1)$ st row of the inverted Vandermonde matrix, $V^{-1}[j]$. So,

$$
a_{k, n}^{[j]}=(-i)^{k} k ! \sum_{m=-j}^{j}\left(V^{-1}[j]\right)_{k+1, j-m+1} \frac{1}{2 \pi} \int_{-\pi}^{+\pi} g_{n}^{[j]}(\theta) e^{i m \theta} d \theta .
$$

Again note that here, unlike the conventions in [1], both the rows and the columns of the Vandermonde matrix and its inverse are indexed as $1,2, \cdots,(2 j+1)$.

Making use of (14a) for $n=0$ gives

$$
a_{k, 0}^{[j]}=(-i)^{k} k ! \sum_{m=-j}^{j}\left(V^{-1}[j]\right)_{k+1, j-m+1}=\delta_{k, 0} .
$$

It is true in general that the entries for any given row of the inverted Vandermonde matrix sum to zero, except for the first row, whose entries sum to one. For instance, see the examples of $V^{-1}$ for $j=1 / 2,1,3 / 2$, and 2 as given in Appendix E. We leave to the interested reader the proof of this elementary fact for any $j$.

Moreover, making use of (14b) for $0<n \in\{k / 2, \cdots, j\}$ gives

$$
\begin{aligned}
b_{k, n}^{[j]} & =(-4)^{n}(-i)^{k} k ! \sum_{m=-j}^{j}\left(V^{-1}[j]\right)_{k+1, j-m+1} \sum_{\ell=n}^{j} \frac{\ell}{n}\left(\begin{array}{c}
\ell+n-1 \\
2 n-1
\end{array}\right) \frac{1}{2 \pi} \int_{-\pi}^{+\pi} \cos (\ell \theta) e^{i m \theta} d \theta \\
& =(-4)^{n}(-i)^{k} k ! \sum_{m=-j}^{j}\left(V^{-1}[j]\right)_{k+1, j-m+1} \sum_{\ell=n}^{j} \frac{\ell}{n}\left(\begin{array}{c}
\ell+n-1 \\
2 n-1
\end{array}\right) \frac{1}{2}\left(\delta_{-\ell, m}+\delta_{\ell, m}\right) \\
& =\frac{1}{2}(-4)^{n}(-i)^{k} k ! \sum_{\ell=n}^{j} \frac{\ell}{n}\left(\begin{array}{c}
\ell+n-1 \\
2 n-1
\end{array}\right) \sum_{m=-j}^{j}\left(V^{-1}[j]\right)_{k+1, j-m+1}\left(\delta_{-\ell, m}+\delta_{\ell, m}\right)
\end{aligned}
$$




$$
\begin{aligned}
& =\frac{1}{2}(-4)^{n}(-i)^{k} k ! \sum_{\ell=n}^{j} \frac{\ell}{n}\left(\begin{array}{c}
\ell+n-1 \\
2 n-1
\end{array}\right)\left(\left(V^{-1}[j]\right)_{k+1, j+\ell+1}+\left(V^{-1}[j]\right)_{k+1, j-\ell+1}\right) \\
& =\frac{(-4)^{n}(-1)^{k / 2} k !}{n} \sum_{\ell=n}^{j} \ell\left(\begin{array}{c}
\ell+n-1 \\
2 n-1
\end{array}\right)\left(V^{-1}[j]\right)_{k+1, j-\ell+1} .
\end{aligned}
$$

In this last relation, the fact that the odd rows of $V^{-1}$ are left $\leftrightarrow$ right column symmetric was used. For instance, again see the examples of $V^{-1}$ for $j=1 / 2,1,3 / 2$, and 2 as given in Appendix E. Also note the even rows of $V^{-1}$ are left $\leftrightarrow$ right column antisymmetric. Again we leave the proof of these elementary facts to the interested reader.

So for even $k \leq 2 j$, upon shifting the summation variable $\ell=j+1-m$, (30) becomes

$$
a_{k, n}^{[j]}=(-4)^{n}(-1)^{k / 2} k ! \sum_{m=1}^{j+1-n}\left(V^{-1}[j]\right)_{k+1, m} \frac{(j+1-m)}{n}\left(\begin{array}{c}
j+n-m \\
2 n-1
\end{array}\right) .
$$

Another Lemma involving the central factorial numbers and the inverted Vandermonde matrix now comes into play, namely,

$$
t(2 n, 2 l)=(2 n) ! 2^{2 l} \sum_{m=1}^{j+1-n}\left(V^{-1}[j]\right)_{2 l+1, m} \frac{(j+1-m)}{n}\left(\begin{array}{c}
j+n-m \\
2 n-1
\end{array}\right),
$$

for $l \in\{1, \cdots, j\}$ and $n \in\{l, \cdots, j\}$. This is established in Appendix C. As a result of this Lemma, (31), and the special case (29), it follows that for even $k \in\{0, \cdots, 2 j\}$,

$$
a_{k, n}^{[j]}=\frac{2^{2 n}}{2^{k}} \frac{k !}{(2 n) !}|t(2 n, k)| \quad \text { for } \quad 2 n \in\{k, \cdots, 2 j\},
$$

where various phases have been cancelled (once more see (A2) in Appendix A).

Note that the only dependence on $j$ is in the upper limit of the sum involved in the series (26), and, correspondingly, on the allowed values of $k$ and $n$ for a given $j$. There is no explicit $j$ dependence in any of the $a_{k, n}^{[j]}$ coefficients.

So for integer $j$ and even $k$,

$$
A_{k}^{[j]}(\theta)=\sum_{n=k / 2}^{j} \frac{2^{2 n}}{2^{k}} \frac{k !}{(2 n) !}|t(2 n, k)| \sin ^{2 n}(\theta / 2)=\frac{k !}{2^{k}} \sum_{m=k}^{2 j} \frac{2^{m}}{m !}|t(m, k)| \sin ^{m}(\theta / 2),
$$

in agreement with (8).

The remaining terms for integer $j$, i.e. the odd $k$ cases in (2), again follow from the fact [1] that odd $k=2 m-1$ coefficients are obtained by differentiating even $k=2 m$ coefficients, as in (6). Thus for integer $j$ this biorthogonal-system-based derivation of (1) is complete.

For semi-integer $j$, a parallel derivation can be constructed using the biorthogonal system of functions involving the odd powers $\sin ^{2 n+1}(\theta / 2)$ and their duals. The details are left as an exercise for the reader.

\section{Conclusion}

The results of Curtright, Fairlie, and Zachos - for rotations expressed as polynomials of spin matrices - were derived here in careful detail, first by elementary methods that rely on the differential relations obtained in [1], and then by methods from the theory of biorthogonal systems, where properties of the central factorial numbers were invoked in both derivations. Either approach confirms the elegant expressions (1) and (2).

Acknowledgement We wish to thank D Fairlie and C Zachos for discussions related to this work. We also thank Jack and Peggy Nichols for their encouragement and support, and especially for their hospitality, while this paper was in preparation. Finally, we thank an anonymous reviewer for pointing out a significant typographical error, and for bringing to our attention references [5] and [7. This work was supported in part by NSF Award PHY-1214521, and in part by a University of Miami Cooper Fellowship. 


\section{References}

[1] T L Curtright, D B Fairlie, and C K Zachos, "A Compact Formula for Rotations as Spin Matrix Polynomials" SIGMA 10 (2014) 084. DOI: 10.3842/SIGMA.2014.084

[2] E P Wigner, Gruppentheorie und ihre Anwendung auf die Quantenmechanik der Atomspektren (1931).

[3] E P Wigner, "On Matrices which Reduce Kroenecker Products of Representations of S. R. Groups" (1938), pp 608-654 in The Collected Works of Eugene Paul Wigner (Volume 1), B R Judd (Editor), G W Mackey (Editor), and J Mehra (Contributor), Springer-Verlag softcover edition (2013).

[4] Y Lehrer-Ilamed,. "On the direct calculations of the representations of the three-dimensional pure rotation group" Proc.Camb.Phil.Soc. 60 (1964) 61-66. DOI: 10.1017/S0305004100037452

[5] R van Wageningen, "Explicit polynomial expressions for finite rotation operators" Nuc.Phys. 60 (1964) 250-263. DOI: 10.1016/0029-5582(64)90660-1

[6] S Weinberg, "Feynman Rules for Any Spin" Phys.Rev. 133 (1964) B1318-B1332 (especially see Appendix A) DOI: 10.1103/PhysRev.133.B1318

[7] T A Weber and S A Williams, "Spin-matrix polynomials and the rotation operator for arbitrary spin" J.Math.Phys. 6 (1965) 1980-1983. DOI: 10.1063/1.1704751

[8] C Zemach, "Use of Angular-Momentum Tensors" Phys. Rev. 140 (1965) B97-B108 (especially see Section IV.3) DOI: 10.1103/PhysRev.140.B97

[9] A J Torruella, "Representations of the three-dimensional rotation group by the direct method" J.Math.Phys. 16 (1975) 1637-1642. DOI: 10.1063/1.522718

[10] R M Young, An Introduction to Non-Harmonic Fourier Series, revised 2nd edition, Academic Press (2001).

[11] T Curtright and L Mezincescu, "Biorthogonal quantum systems" J.Math.Phys. 48 (2007) 092106. DOI: $10.1063 / 1.2196243$

[12] T Curtright, L Mezincescu, and D Schuster, "Supersymmetric biorthogonal quantum systems" J.Math.Phys. 48 (2007) 092108. DOI: $10.1063 / 1.2191367$

[13] D C Brody, "Biorthogonal Quantum Mechanics" J.Phys.A:Math.Theor. 47 035305. DOI: 10.1088/1751$8113 / 47 / 3 / 035305$

[14] J Riordan, Combinatorial Identities, Wiley (1968). (Also see http://oeis.org/A008955 and additional references therein.)

[15] P L Butzer, M Schmidt, E L Stark, and L Vogt, "Central Factorial Numbers: Their main properties and some applications" Numerical Functional Analysis and Optimization 10 (1989) 419-488.

[16] T L Curtright, D B Fairlie, and C K Zachos, A Concise Treatise on Quantum Mechanics in Phase Space, World Scientific and Imperial College Press (2014).

[17] H-W Lee, "Theory and application of the quantum phase-space distribution functions" Physics Reports 259 (1995) 147-211. DOI: 10.1016/0370-1573(95)00007-4 


\section{Appendix A: Central factorial numbers}

For historical reasons, central factorial numbers are defined as the coefficients in simple polynomials [14, 15. They can be either positive or negative, but only their absolute values are needed for the coefficients of the spin matrix expansions in the main text. Moreover, $t$ (even, even) are integers, but $t$ (odd,odd) are not integers, and $t($ odd , even $)=0=t($ even, odd $)$. So the even and odd cases are best handled separately.

By definition and as elementary consequences thereof (cf. http://oeis.org/A182867),

$$
\begin{gathered}
\prod_{l=0}^{m-1}\left(x^{2}-l^{2}\right)=\sum_{k=1}^{m} t(2 m, 2 k) x^{2 k} \\
t(2 m, 2 k)=(-1)^{m-k}|t(2 m, 2 k)|=\left.(-1)^{m-k} \frac{1}{k !} \frac{d^{k}}{d z^{k}} \prod_{l=0}^{m-1}\left(z+l^{2}\right)\right|_{z=0},
\end{gathered}
$$

as well as (cf. http://oeis.org/A008956)

$$
\begin{gathered}
x \prod_{l=0}^{m-1}\left(x^{2}-\left(l+\frac{1}{2}\right)^{2}\right)=\sum_{k=0}^{m} t(2 m+1,2 k+1) x^{2 k+1} \\
t(2 m+1,2 k+1)=(-1)^{m-k}|t(2 m+1,2 k+1)|=\left.(-1)^{m-k} \frac{1}{k !} \frac{d^{k}}{d z^{k}} \prod_{l=0}^{m-1}\left(z+\left(l+\frac{1}{2}\right)^{2}\right)\right|_{z=0} .
\end{gathered}
$$

\section{Appendix B: A useful lemma}

Proof of the Lemma:

$$
(2 \hat{\boldsymbol{n}} \cdot \boldsymbol{J})^{2 j+1}=-\sum_{k=0}^{2 j} 2^{1+2 j-k} \times t(2+2 j, 1+k) \times(2 \hat{\boldsymbol{n}} \cdot \boldsymbol{J})^{k},
$$

where $t(m, n)$ are the central factorial numbers, defined in Appendix A. In a basis where $2 \hat{\boldsymbol{n}} \cdot \boldsymbol{J}$ is diagonal, (B1) reduces to a matrix equation,

$$
\left(\begin{array}{c}
(2 j)^{2 j+1} \\
(2 j-2)^{2 j+1} \\
\vdots \\
(-2 j+2)^{2 j+1} \\
(-2 j)^{2 j+1}
\end{array}\right)=-2^{1+2 j} \times V[j]\left(\begin{array}{c}
t(2+2 j, 1) \\
\frac{1}{2} t(2+2 j, 2) \\
\vdots \\
\frac{1}{2^{2 j-1}} t(2+2 j, 2 j) \\
\frac{1}{2^{2 j}} t(2+2 j, 1+2 j)
\end{array}\right),
$$

where the Vandermonde matrix for spin $j$ is defined by

$$
V[j]=\left(\begin{array}{ccccc}
1 & 2 j & (2 j)^{2} & \cdots & (2 j)^{2 j} \\
1 & 2 j-2 & (2 j-2)^{2} & \cdots & (2 j-2)^{2 j} \\
\vdots & \vdots & \vdots & \ddots & \vdots \\
1 & -2 j & (-2 j)^{2} & \cdots & (-2 j)^{2 j}
\end{array}\right) .
$$

So, consider the $k$ th row on the RHS of (B2):

$$
-\frac{2^{1+2 j}}{(j+1-k)} \times\left(\begin{array}{c}
t(2+2 j, 1)(j+1-k)+t(2+2 j, 2)(j+1-k)^{2}+t(2+2 j, 3)(j+1-k)^{3}+\cdots \\
+t(2+2 j, 2 j)(j+1-k)^{2 j-1}+t(2+2 j, 1+2 j)(j+1-k)^{2 j}
\end{array}\right) .
$$

If $j$ is an integer, then $t(2+2 j, o d d)=0$, and this $k$ th row becomes

$$
\begin{aligned}
& -\frac{2^{1+2 j}}{(j+1-k)} \times\left(\begin{array}{c}
t(2+2 j, 2)(j+1-k)^{2}+t(2+2 j, 4)(j+1-k)^{4}+\cdots \\
+t(2+2 j, 2 j-2)(j+1-k)^{2 j-2}+t(2+2 j, 2 j)(j+1-k)^{2 j} \\
+t(2+2 j, 2 j+2)(j+1-k)^{2 j+2}-t(2+2 j, 2 j+2)(j+1-k)^{2 j+2}
\end{array}\right) \\
& =-\frac{2^{1+2 j}}{(j+1-k)} \times\left(\prod_{l=0}^{j}\left((j+1-k)^{2}-l^{2}\right)-t(2+2 j, 2 j+2) \times(j+1-k)^{2 j+2}\right),
\end{aligned}
$$


where the $t(2+2 j, 2 j+2)$ term was added and subtracted to obtain the complete sum on the RHS of (A1), for $m=j+1$ and $x=j+1-k$, and then that sum was replaced with the product on the LHS of (A1). But the product evaluates to zero because one of the terms in the product always vanishes for $k \geq 1$, and therefore the $k$ th row on the RHS of (B2) is

$$
t(2+2 j, 2+2 j) \times(2 j+2-2 k)^{2 j+1}=(2 j+2-2 k)^{2 j+1},
$$

since $t(n, n)=1$, Thus we obtain the $k$ th row on the LHS of (B2), and the Lemma is proven for integer $j$.

If $j$ is semi-integer, a corresponding proof goes through just as easily, upon using (A4).

\section{Appendix C: Another useful lemma}

Proof of the Lemma:

$$
t(2 n, 2 l)=(2 n) ! 2^{2 l} \sum_{m=1}^{j+1-n}\left(V^{-1}[j]\right)_{2 l+1, m} \frac{(j+1-m)}{n}\left(\begin{array}{c}
j+n-m \\
2 n-1
\end{array}\right),
$$

for integer $j$, for $l \in\{1, \cdots, j\}$, and for $0<n \in\{l, \cdots, j\}$, where $t(m, k)$ are the central factorial numbers defined in Appendix A. Following steps similar to those used in Appendix B leads immediately to the result:

$$
\sum_{q=1}^{2 j+1}(V[j])_{m, q} \frac{1}{2^{q}} t(2 n, q-1)=\sum_{l=1}^{j}(V[j])_{m, 2 l+1} \frac{1}{2^{2 l+1}} t(2 n, 2 l)=\frac{1}{2} \prod_{k=0}^{n-1}\left((j+1-m)^{2}-k^{2}\right) .
$$

It then follows from left-multiplication by $V^{-1}[j]$ that

$$
\frac{1}{2^{2 l+1}} t(2 n, 2 l)=\frac{1}{2} \sum_{m=1}^{2 j+1}\left(V^{-1}[j]\right)_{2 l+1, m} \prod_{k=0}^{n-1}\left((j+1-m)^{2}-k^{2}\right) .
$$

These are the non-vanishing cases of interest. But then,

$$
\prod_{k=0}^{n-1}\left((j+1-m)^{2}-k^{2}\right)=\left\{\begin{array}{ccc}
\frac{(j+1-m)(j+n-m) !}{(j+1-n-m) !} & \text { for } & 1 \leq m \leq j+1-n \\
0 & \text { for } & j+2-n \leq m \leq j+n \\
\frac{(m-1-j)(m+n-j-2) !}{(m-n-j-1) !} & \text { for } & j+1+n \leq m \leq 2 j+1
\end{array} .\right.
$$

Thus we obtain

$$
\begin{aligned}
t(2 n, 2 l) & =2^{2 l} \sum_{m=1}^{j+1-n}\left(V^{-1}[j]\right)_{2 l+1, m} \frac{(j+1-m)(j+n-m) !}{(j+1-n-m) !} \\
& +2^{2 l} \sum_{m=j+1+n}^{2 j+1}\left(V^{-1}[j]\right)_{2 l+1, m} \frac{(m-1-j)(m+n-j-2) !}{(m-n-j-1) !} .
\end{aligned}
$$

However, the two sums on the RHS of (C5) are equal. In fact, the summands are equal term-by-term, as a consequence of the left $\leftrightarrow$ right column symmetry of the odd rows of $V^{-1}[j]$. That is to say,

$$
\begin{gathered}
\left.\frac{(j+1-k)(j+n-k) !}{(j+1-n-k) !}\right|_{k=m}=\left.\frac{(k-1-j)(k+n-j-2) !}{(k-n-j-1) !}\right|_{k=2 j+2-m}, \\
\left(V^{-1}[j]\right)_{2 l+1, m}=\left(V^{-1}[j]\right)_{2 l+1,2 j+2-m},
\end{gathered}
$$

for $m=1, \cdots, j+1$. The final result is then the sought-for relation:

$$
\begin{aligned}
t(2 n, 2 l) & =2 \times 2^{2 l} \sum_{m=1}^{j+1-n}\left(V^{-1}[j]\right)_{2 l+1, m} \frac{(j+1-m)(j+n-m) !}{(j+1-n-m) !} \\
& \equiv(2 n) ! 2^{2 l} \sum_{m=1}^{j+1-n}\left(V^{-1}[j]\right)_{2 l+1, m} \frac{(j+1-m)}{n}\left(\begin{array}{c}
j+n-m \\
2 n-1
\end{array}\right) .
\end{aligned}
$$




\section{Appendix D: Finite biorthogonal systems of functions}

Here are more details about the finite biorthogonal systems of functions described in the main text, constructed such that

$$
\delta_{m, n}=\frac{1}{2 \pi} \int_{-\pi}^{+\pi} g_{m}^{[j]}(\theta) f_{n}^{[j]}(\theta) d \theta .
$$

Consider first the even powers of $\sin (\theta / 2)$ and their duals. The biorthogonal system in this situation is given by the following Table.

Table 1 For $j \in\{0,1,2,3, \cdots\}$ :

\begin{tabular}{|c|c|c|}
\hline Function $f_{n}^{[j]}$ & $n$ & Dual Function $g_{n}^{[j]}$ \\
\hline 1 & 0 & {$[1+2 \cos (\theta)+2 \cos (2 \theta)+2 \cos (3 \theta)+2 \cos (4 \theta)+2 \cos (5 \theta)+\cdots+2 \cos (j \theta)]$} \\
\hline $\sin ^{2}(\theta / 2)$ & 1 & $-4\left[\cos (\theta)+4 \cos (2 \theta)+9 \cos (3 \theta)+16 \cos (4 \theta)+25 \cos (5 \theta)+\cdots+j^{2} \cos (j \theta)\right]$ \\
\hline $\sin ^{4}(\theta / 2)$ & 2 & $16\left[\cos (2 \theta)+6 \cos (3 \theta)+20 \cos (4 \theta)+50 \cos (5 \theta)+\cdots+\frac{1}{2}\left(\begin{array}{c}j+1 \\
3\end{array}\right) j \cos (j \theta)\right]$ \\
\hline $\sin ^{6}(\theta / 2)$ & 3 & $-64\left[\cos (3 \theta)+8 \cos (4 \theta)+35 \cos (5 \theta)+112 \cos (6 \theta)+\cdots+\frac{1}{3}\left(\begin{array}{c}j+2 \\
5\end{array}\right) j \cos (j \theta)\right]$ \\
\hline $\sin ^{8}(\theta / 2)$ & 4 & $256\left[\cos (4 \theta)+10 \cos (5 \theta)+54 \cos (6 \theta)+210 \cos (7 \theta)+\cdots+\frac{1}{4}\left(\begin{array}{c}j+3 \\
7\end{array}\right) j \cos (j \theta)\right]$ \\
\hline $\sin ^{10}(\theta / 2)$ & 5 & $-1024\left[\cos (5 \theta)+12 \cos (6 \theta)+77 \cos (7 \theta)+352 \cos (8 \theta)+\cdots+\frac{1}{5}\left(\begin{array}{c}j+4 \\
9\end{array}\right) j \cos (j \theta)\right]$ \\
\hline$\vdots$ & $\vdots$ & $\begin{array}{lll}\vdots & \vdots & \vdots\end{array}$ \\
\hline $\sin ^{2 j-6}(\theta / 2)$ & $j-3$ & $(-4)^{j-3}\left[\cos ((j-3) \theta)+2(j-2) \cos ((j-2) \theta)+\cdots+\frac{1}{j-3}\left(\begin{array}{c}2 j-4 \\
2 j-7\end{array}\right) j \cos (j \theta)\right]$ \\
\hline $\sin ^{2 j-4}(\theta / 2)$ & $j-2$ & $(-4)^{j-2}[\cos ((j-2) \theta)+2(j-1) \cos ((j-1) \theta)+(2 j-3) j \cos (j \theta)]$ \\
\hline $\sin ^{2 j-2}(\theta / 2)$ & $j-1$ & $(-4)^{j-1}[\cos ((j-1) \theta)+2 j \cos (j \theta)]$ \\
\hline $\sin ^{2 j}(\theta / 2)$ & $j$ & $(-4)^{j}[\cos (j \theta)]$ \\
\hline
\end{tabular}

Consider next the odd powers of $\sin (\theta / 2)$ and their duals. As noted in the main text, a biorthogonal system for this situation may be obtained from the system involving even powers of $\sin (\theta / 2)$ just by moving a single factor of $\sin (\theta / 2)$ from the functions to the dual functions. For application to the spin matrix expansion, consider semi-integer $j$. The $j+1 / 2$ lowest odd powers of $\sin (\theta / 2)$, beginning with $\sin (\theta / 2)$ and ending with $\sin ^{2 j}(\theta / 2)$, again constitute one half of a biorthogonal system of functions. The functions and their duals are given by the following Table.

Table 2 For $j \in\left\{\frac{1}{2}, \frac{3}{2}, \frac{5}{2}, \frac{7}{2}, \cdots\right\}$ :

\begin{tabular}{|c|c|c|}
\hline Function $f_{n}^{[j]}$ & $n$ & Dual Function $g_{n}^{[j]}$ \\
\hline $\sin (\theta / 2)$ & 1 & $-4 \sin (\theta / 2)\left[\cos (\theta)+4 \cos (2 \theta)+9 \cos (3 \theta)+16 \cos (4 \theta)+25 \cos (5 \theta)+\cdots+\left(j+\frac{1}{2}\right)^{2} \cos \left(\left(j+\frac{1}{2}\right) \theta\right)\right]$ \\
\hline $\sin ^{3}(\theta / 2)$ & 2 & $16 \sin (\theta / 2)\left[\cos (2 \theta)+6 \cos (3 \theta)+20 \cos (4 \theta)+50 \cos (5 \theta)+\cdots+\frac{1}{2}\left(\begin{array}{c}j+3 / 2 \\
3\end{array}\right)\left(j+\frac{1}{2}\right) \cos \left(\left(j+\frac{1}{2}\right) \theta\right)\right]$ \\
\hline $\sin ^{5}(\theta / 2)$ & 3 & $-64 \sin (\theta / 2)\left[\cos (3 \theta)+8 \cos (4 \theta)+35 \cos (5 \theta)+112 \cos (6 \theta)+\cdots+\frac{1}{3}\left({ }_{5}^{j+5 / 2}\right)\left(j+\frac{1}{2}\right) \cos \left(\left(j+\frac{1}{2}\right) \theta\right)\right]$ \\
\hline $\sin ^{7}(\theta / 2)$ & 4 & $256 \sin (\theta / 2)\left[\cos (4 \theta)+10 \cos (5 \theta)+54 \cos (6 \theta)+210 \cos (7 \theta)+\cdots+\frac{1}{4}\left({ }_{7}^{j+7 / 2}\right)\left(j+\frac{1}{2}\right) \cos \left(\left(j+\frac{1}{2}\right) \theta\right)\right]$ \\
\hline $\sin ^{9}(\theta / 2)$ & 5 & $-1024 \sin (\theta / 2)\left[\cos (5 \theta)+12 \cos (6 \theta)+77 \cos (7 \theta)+352 \cos (8 \theta)+\cdots+\frac{1}{5}\left({ }_{9}^{j+9 / 2}\right)\left(j+\frac{1}{2}\right) \cos \left(\left(j+\frac{1}{2}\right) \theta\right)\right.$ \\
\hline $\sin ^{11}(\theta / 2)$ & 6 & $4096 \sin (\theta / 2)\left[\cos (6 \theta)+14 \cos (7 \theta)+104 \cos (8 \theta)+546 \cos (9 \theta)+\cdots+\frac{1}{6}{ }_{11}^{j+11 / 2}\right)\left(j+\frac{1}{2}\right) \cos \left(\left(j+\frac{1}{2}\right) \theta\right)$ \\
\hline$\vdots$ & $\vdots$ & $\begin{array}{lll}\vdots & \vdots & \vdots\end{array}$ \\
\hline $\sin ^{2 j-6}(\theta / 2)$ & $j-\frac{5}{2}$ & $(-4)^{j-\frac{5}{2}} \sin (\theta / 2)\left[\cos \left(\left(j-\frac{5}{2}\right) \theta\right)+2\left(j-\frac{3}{2}\right) \cos \left(\left(j-\frac{3}{2}\right) \theta\right)+\cdots+\frac{1}{j-\frac{5}{2}}\left(\begin{array}{c}2 j-3 \\
2 j-6\end{array}\right)\left(j+\frac{1}{2}\right) \cos \left(\left(j+\frac{1}{2}\right) \theta\right)\right]$ \\
\hline $\sin ^{2 j-4}(\theta / 2)$ & $j-\frac{3}{2}$ & $(-4)^{j-\frac{3}{2}} \sin (\theta / 2)\left[\cos \left(\left(j-\frac{3}{2}\right) \theta\right)+2\left(j-\frac{1}{2}\right) \cos \left(\left(j-\frac{1}{2}\right) \theta\right)+(2 j-2)\left(j+\frac{1}{2}\right) \cos \left(\left(j+\frac{1}{2}\right) \theta\right)\right]$ \\
\hline $\sin ^{2 j-2}(\theta / 2)$ & $j-\frac{1}{2}$ & $(-4)^{j-\frac{1}{2}} \sin (\theta / 2)\left[\cos \left(\left(j-\frac{1}{2}\right) \theta\right)+2\left(j+\frac{1}{2}\right) \cos \left(\left(j+\frac{1}{2}\right) \theta\right)\right]$ \\
\hline $\sin ^{2 j}(\theta / 2)$ & $j+\frac{1}{2}$ & $(-4)^{j+\frac{1}{2}} \sin (\theta / 2)\left[\cos \left(\left(j+\frac{1}{2}\right) \theta\right)\right]$ \\
\hline
\end{tabular}


As stated in the main text, these two systems may be combined into a larger one, involving both even and odd powers of $\sin (\theta / 2)$, without modification of the dual functions. An explicit Table for the enlarged system may be obtained just by interlacing the rows of Tables 1 and 2 .

The procedure to obtain the dual functions is straightforward. For example, start at the highest power of $\sin (\theta / 2)$, namely, $\sin ^{2 j}(\theta / 2)$, as given in the last row of Table 1 , for which an obvious dual function is the Chebyshev polynomial $\cos (j \theta)$ with coefficient $(-4)^{j}$, as is easily verified. Note that $\cos (j \theta)$ is manifestly orthogonal to all powers $\sin ^{2 n}(\theta / 2)$ with $n<j$. Now consider the next to highest power, namely, $\sin ^{2 j-2}(\theta / 2)$, as given in the next-to-last row of Table 1 . The first term of its dual function is just what it would be if one were considering the biorthogonal system with $j$ reduced by 1 , namely, $\cos ((j-1) \theta)$ with coefficient $(-4)^{j-1}$. But this term alone is not orthogonal to $\sin ^{2 j}(\theta / 2)$, so one must add the higher harmonic $\cos (j \theta)$ with coefficient to achieve the desired orthogonality. The higher harmonic is clearly orthogonal to $\sin ^{2 j-2}(\theta / 2)$ and all lower powers of $\sin ^{2}(\theta / 2)$, so it does not contribute to the orthonormalization integral for $\sin ^{2 j-2}(\theta / 2)$. And so it goes. The results for the duals of the lower powers of $\sin ^{2}(\theta / 2)$ are iterated series of terms where, in any particular row of Table 1, all but the highest harmonic are given by the same terms as appear in the subsequent row of the Table (after replacing $j$ in the earlier row by $j+1$ in the later row), and where the coefficient of the highest harmonic in the earlier row, namely, $\cos (j \theta)$, is determined by requiring orthogonality to $\sin ^{2 j}(\theta / 2)$.

To carry out the construction of the dual functions, the following integral is useful:

$$
\int_{-\pi}^{\pi} \cos (m \theta) \sin ^{2 j}(\theta / 2) d \theta=\frac{2 \pi(-1)^{m}}{4^{j}}\left(\begin{array}{c}
2 j \\
j+m
\end{array}\right) \text { for } \quad 0 \leq m \leq j .
$$

Note also the following generating functions for the coefficients inside the square brackets in the various rows of Table 1:

$$
\begin{aligned}
& \frac{1+x}{1-x}=1+2 x+2 x^{2}+2 x^{3}+2 x^{4}+2 x^{5}+2 x^{6}+2 x^{7}+O\left(x^{8}\right), \\
& \frac{1+x}{(1-x)^{3}}=1+4 x+9 x^{2}+16 x^{3}+25 x^{4}+36 x^{5}+49 x^{6}+64 x^{7}+O\left(x^{8}\right), \\
& \frac{1+x}{(1-x)^{5}}=1+6 x+20 x^{2}+50 x^{3}+105 x^{4}+196 x^{5}+336 x^{6}+540 x^{7}+O\left(x^{8}\right), \\
& \frac{1+x}{(1-x)^{7}}=1+8 x+35 x^{2}+112 x^{3}+294 x^{4}+672 x^{5}+1386 x^{6}+2640 x^{7}+O\left(x^{8}\right), \\
& \frac{1+x}{(1-x)^{9}}=1+10 x+54 x^{2}+210 x^{3}+660 x^{4}+1782 x^{5}+4290 x^{6}+9438 x^{7}+O\left(x^{8}\right), \\
& \frac{1+x}{(1-x)^{11}}=1+12 x+77 x^{2}+352 x^{3}+1287 x^{4}+4004 x^{5}+11011 x^{6}+27456 x^{7}+O\left(x^{8}\right), \\
& \frac{1+x}{(1-x)^{13}}=1+14 x+104 x^{2}+546 x^{3}+2275 x^{4}+8008 x^{5}+24752 x^{6}+68952 x^{7}+O\left(x^{8}\right), \\
& \frac{1+x}{(1-x)^{2 j-5}}=1+2(j-2) x+(2 j-5)(j-1) x^{2}+\frac{2}{3}(2 j-5)(j-2) j x^{3}+O\left(x^{4}\right), \\
& \frac{1+x}{(1-x)^{2 j-3}}=1+2(j-1) x+(2 j-3) j x^{2}+O\left(x^{3}\right), \\
& \frac{1+x}{(1-x)^{2 j-1}}=1+2 j x+O\left(x^{2}\right), \\
& \frac{1+x}{(1-x)^{2 j+1}}=1+O(x) .
\end{aligned}
$$

These generating functions are a direct consequence of (16). Finally, note of course that $\frac{1}{2}\left(\begin{array}{c}j+1 \\ 3\end{array}\right) j=\frac{1}{12}\left(j^{2}-1\right) j^{2}$, and $\frac{1}{j-3}\left(\begin{array}{c}2 j-4 \\ 2 j-7\end{array}\right) j=\frac{2}{3}(2 j-5)(j-2) j$, etc., but in the Tables these are expressed as binomial coefficients to display more clearly the general pattern. 


\section{Appendix E: Biorthogonal matrix examples}

Here are more details about the biorthogonal systems of spin matrices described in the main text, for $j=$ $1 / 2,1,3 / 2$, and 2 .

Spin $j=1 / 2$ is deceptively simple. The independent powers of the spin matrix are

$$
S^{0}=\left(\begin{array}{ll}
1 & 0 \\
0 & 1
\end{array}\right), \quad S^{1}=\left(\begin{array}{cc}
1 & 0 \\
0 & -1
\end{array}\right),
$$

and the corresponding trace-orthonormal dual matrices are the same up to a normalization factor. (NB This is not true for any other $j$.)

$$
T_{0}=\frac{1}{2}\left(\begin{array}{ll}
1 & 0 \\
0 & 1
\end{array}\right), \quad T_{1}=\frac{1}{2}\left(\begin{array}{cc}
1 & 0 \\
0 & -1
\end{array}\right), \quad \text { i.e. } \quad V^{-1}=\frac{1}{2}\left(\begin{array}{cc}
1 & 1 \\
1 & -1
\end{array}\right), \quad G=\frac{1}{2}\left(\begin{array}{ll}
1 & 0 \\
0 & 1
\end{array}\right) .
$$

Spin $j=1$ is a more interesting example. The independent powers of the spin matrix are given by

$$
S^{0}=\left(\begin{array}{lll}
1 & 0 & 0 \\
0 & 1 & 0 \\
0 & 0 & 1
\end{array}\right), \quad S^{1}=\left(\begin{array}{ccc}
2 & 0 & 0 \\
0 & 0 & 0 \\
0 & 0 & -2
\end{array}\right), \quad S^{2}=\left(\begin{array}{ccc}
4 & 0 & 0 \\
0 & 0 & 0 \\
0 & 0 & 4
\end{array}\right)
$$

and the corresponding trace-orthonormal dual matrices, as well as $V^{-1}$ and $G$, are given by

$$
\begin{aligned}
T_{0} & =\left(\begin{array}{lll}
0 & 0 & 0 \\
0 & 1 & 0 \\
0 & 0 & 0
\end{array}\right), \quad T_{1}=\frac{1}{4}\left(\begin{array}{ccc}
1 & 0 & 0 \\
0 & 0 & 0 \\
0 & 0 & -1
\end{array}\right), T_{3}=\frac{1}{8}\left(\begin{array}{ccc}
1 & 0 & 0 \\
0 & -2 & 0 \\
0 & 0 & 1
\end{array}\right), \\
V^{-1} & =\frac{1}{8}\left(\begin{array}{ccc}
0 & 8 & 0 \\
2 & 0 & -2 \\
1 & -2 & 1
\end{array}\right), \quad G=\frac{1}{64}\left(\begin{array}{ccc}
5 & -2 & -3 \\
-2 & 68 & -2 \\
-3 & -2 & 5
\end{array}\right) .
\end{aligned}
$$

Spin $j=3 / 2$ is also interesting. The independent powers of the spin matrix are given by

$$
S^{0}=\left(\begin{array}{llll}
1 & 0 & 0 & 0 \\
0 & 1 & 0 & 0 \\
0 & 0 & 1 & 0 \\
0 & 0 & 0 & 1
\end{array}\right), \quad S^{1}=\left(\begin{array}{cccc}
3 & 0 & 0 & 0 \\
0 & 1 & 0 & 0 \\
0 & 0 & -1 & 0 \\
0 & 0 & 0 & -3
\end{array}\right), \quad S^{2}=\left(\begin{array}{llll}
9 & 0 & 0 & 0 \\
0 & 1 & 0 & 0 \\
0 & 0 & 1 & 0 \\
0 & 0 & 0 & 9
\end{array}\right), \quad S^{3}=\left(\begin{array}{cccc}
27 & 0 & 0 & 0 \\
0 & 1 & 0 & 0 \\
0 & 0 & -1 & 0 \\
0 & 0 & 0 & -27
\end{array}\right),
$$

and the corresponding trace-orthonormal dual matrices, as well as $V^{-1}$ and $G$, are given by

$$
\begin{aligned}
& T_{0}=\frac{1}{16}\left(\begin{array}{cccc}
-1 & 0 & 0 & 0 \\
0 & 9 & 0 & 0 \\
0 & 0 & 9 & 0 \\
0 & 0 & 0 & -1
\end{array}\right), \quad T_{1}=\frac{1}{48}\left(\begin{array}{cccc}
-1 & 0 & 0 & 0 \\
0 & 27 & 0 & 0 \\
0 & 0 & -27 & 0 \\
0 & 0 & 0 & 1
\end{array}\right), \quad T_{2}=\frac{1}{16}\left(\begin{array}{cccc}
1 & 0 & 0 & 0 \\
0 & -1 & 0 & 0 \\
0 & 0 & -1 & 0 \\
0 & 0 & 0 & 1
\end{array}\right) \\
& T_{3}=\frac{1}{48}\left(\begin{array}{cccc}
1 & 0 & 0 & 0 \\
0 & -3 & 0 & 0 \\
0 & 0 & 3 & 0 \\
0 & 0 & 0 & -1
\end{array}\right), \quad V^{-1}=\frac{1}{48}\left(\begin{array}{cccc}
-3 & 27 & 27 & -3 \\
-1 & 27 & -27 & 1 \\
3 & -3 & -3 & 3 \\
1 & -3 & 3 & -1
\end{array}\right) \quad . \quad G=\frac{1}{48^{2}}\left(\begin{array}{cccc}
20 & -120 & -60 & 16 \\
-120 & 1476 & 0 & -60 \\
-60 & 0 & 1476 & -120 \\
16 & -60 & -120 & 20
\end{array}\right)
\end{aligned}
$$

Spin $j=2$ helps to establish the general pattern.

$$
S^{m}=\left(\begin{array}{ccccc}
4^{m} & 0 & 0 & 0 & 0 \\
0 & 2^{m} & 0 & 0 & 0 \\
0 & 0 & 0 & 0 & 0 \\
0 & 0 & 0 & (-2)^{m} & 0 \\
0 & 0 & 0 & 0 & (-4)^{m}
\end{array}\right), T_{0}=\left(\begin{array}{ccccc}
0 & 0 & 0 & 0 & 0 \\
0 & 0 & 0 & 0 & 0 \\
0 & 0 & 1 & 0 & 0 \\
0 & 0 & 0 & 0 & 0 \\
0 & 0 & 0 & 0 & 0
\end{array}\right), T_{1}=\frac{1}{24}\left(\begin{array}{ccccc}
-1 & 0 & 0 & 0 & 0 \\
0 & 8 & 0 & 0 & 0 \\
0 & 0 & 0 & 0 & 0 \\
0 & 0 & 0 & -8 & 0 \\
0 & 0 & 0 & 0 & 1
\end{array}\right),
$$




$$
\begin{aligned}
& T_{2}=\frac{1}{96}\left(\begin{array}{ccccc}
-1 & 0 & 0 & 0 & 0 \\
0 & 16 & 0 & 0 & 0 \\
0 & 0 & -30 & 0 & 0 \\
0 & 0 & 0 & 16 & 0 \\
0 & 0 & 0 & 0 & -1
\end{array}\right), \quad T_{3}=\frac{1}{96}\left(\begin{array}{ccccc}
1 & 0 & 0 & 0 & 0 \\
0 & -2 & 0 & 0 & 0 \\
0 & 0 & 0 & 0 & 0 \\
0 & 0 & 0 & 2 & 0 \\
0 & 0 & 0 & 0 & -1
\end{array}\right), \quad T_{4}=\frac{1}{384}\left(\begin{array}{ccccc}
1 & 0 & 0 & 0 & 0 \\
0 & -4 & 0 & 0 & 0 \\
0 & 0 & 6 & 0 & 0 \\
0 & 0 & 0 & -4 & 0 \\
0 & 0 & 0 & 0 & 1
\end{array}\right), \\
& V^{-1}=\frac{1}{384}\left(\begin{array}{ccccc}
0 & 0 & 384 & 0 & 0 \\
-16 & 128 & 0 & -128 & 16 \\
-4 & 64 & -120 & 64 & -4 \\
4 & -8 & 0 & 8 & -4 \\
1 & -4 & 6 & -4 & 1
\end{array}\right) \quad, \quad G=\frac{1}{384^{2}}\left(\begin{array}{ccccc}
289 & -2340 & 486 & 1820 & -255 \\
-2340 & 20560 & -7704 & -12336 & 1820 \\
486 & -7704 & 161892 & -7704 & 486 \\
1820 & -12336 & -7704 & 20560 & -2340 \\
-255 & 1820 & 486 & -2340 & 289
\end{array}\right)
\end{aligned}
$$

For the discussion in the text about the $S$ and $T$ matrix biorthogonality expressed as a trace, and the related discussion in the following Appendix $\mathrm{F}$, it may also be helpful for the reader to consider the $B$ and $P$ matrices for these spins, especially to check (F3) and (F4).

For example, for $\operatorname{spin} j=1$,

$$
\begin{aligned}
B & =\left(\begin{array}{l}
1 \\
1 \\
1
\end{array}\right)\left(\begin{array}{lll}
1 & 1 & 1
\end{array}\right)=\left(\begin{array}{lll}
1 & 1 & 1 \\
1 & 1 & 1 \\
1 & 1 & 1
\end{array}\right), \\
P & =\left(\begin{array}{lll}
1 & 0 & 0 \\
0 & 0 & 0 \\
0 & 0 & 0
\end{array}\right)=\frac{1}{64}\left(\begin{array}{ccc}
0 & 8 & 0 \\
2 & 0 & -2 \\
1 & -2 & 1
\end{array}\right)\left(\begin{array}{lll}
1 & 1 & 1 \\
1 & 1 & 1 \\
1 & 1 & 1
\end{array}\right)\left(\begin{array}{ccc}
0 & 2 & 1 \\
8 & 0 & -2 \\
0 & -2 & 1
\end{array}\right) .
\end{aligned}
$$

For this example, eigenvalues and a particular choice of orthogonal eigenvectors for $B$ are given by

$$
B\left(\begin{array}{l}
1 \\
1 \\
1
\end{array}\right)=3\left(\begin{array}{l}
1 \\
1 \\
1
\end{array}\right), \quad \text { and } B\left(\begin{array}{c}
2 \\
-1 \\
-1
\end{array}\right)=B\left(\begin{array}{c}
0 \\
1 \\
-1
\end{array}\right)=0 \text {. }
$$

For $\operatorname{spin} j=3 / 2$,

$$
\begin{aligned}
B & =\left(\begin{array}{l}
1 \\
1 \\
1 \\
1
\end{array}\right)\left(\begin{array}{llll}
1 & 1 & 1 & 1
\end{array}\right)=\left(\begin{array}{llll}
1 & 1 & 1 & 1 \\
1 & 1 & 1 & 1 \\
1 & 1 & 1 & 1 \\
1 & 1 & 1 & 1
\end{array}\right), \\
P & =\left(\begin{array}{llll}
1 & 0 & 0 & 0 \\
0 & 0 & 0 & 0 \\
0 & 0 & 0 & 0 \\
0 & 0 & 0 & 0
\end{array}\right)=\frac{1}{2304}\left(\begin{array}{cccc}
-3 & 27 & 27 & -3 \\
-1 & 27 & -27 & 1 \\
3 & -3 & -3 & 3 \\
1 & -3 & 3 & -1
\end{array}\right)\left(\begin{array}{llll}
1 & 1 & 1 & 1 \\
1 & 1 & 1 & 1 \\
1 & 1 & 1 & 1 \\
1 & 1 & 1 & 1
\end{array}\right)\left(\begin{array}{cccc}
-3 & -1 & 3 & 1 \\
27 & 27 & -3 & -3 \\
27 & -27 & -3 & 3 \\
-3 & 1 & 3 & -1
\end{array}\right) .
\end{aligned}
$$

Particular orthogonal eigenvectors and eigenvalues of $B$ are now given by

$$
B\left(\begin{array}{l}
1 \\
1 \\
1 \\
1
\end{array}\right)=4\left(\begin{array}{l}
1 \\
1 \\
1 \\
1
\end{array}\right) \text {, and } B\left(\begin{array}{c}
1 \\
-1 \\
0 \\
0
\end{array}\right)=B\left(\begin{array}{c}
0 \\
0 \\
1 \\
-1
\end{array}\right)=B\left(\begin{array}{c}
1 \\
1 \\
-1 \\
-1
\end{array}\right)=0 .
$$

The generalization to other $j$ is straightforward. 


\section{Appendix F: Trace of a matrix star product}

Let us pursue the business of writing the matrix biorthonormality in the main text as a trace relation.

$$
\delta_{m, n}=\operatorname{Trace}\left(G S^{n} B S^{m}\right),
$$

where, as before, $B$ is a singular matrix with all entries equal to 1 . We have cycled the matrices in (24) into the ordering shown in (F1) to emphasize a possible analogy to distributions integrated over phase space [16]. The second ordering suggests more clearly which of $B \& G$ corresponds to a "measure" (note that $G$ is nonsingular) and which to a "star product" (once again, note that $B$ is singular).

Moreover, for any spin $j$ the matrix $B$ is obtained by acting on the projector

$$
P=\left(\begin{array}{cccc}
1 & 0 & 0 & \cdots \\
0 & 0 & 0 & \cdots \\
0 & 0 & 0 & \cdots \\
\vdots & \vdots & \vdots & \ddots
\end{array}\right)
$$

with the Vandermonde matrix and its transpose:

$$
B=V[j] P V[j]^{\text {transpose }}=\left(\begin{array}{cccc}
1 & 1 & 1 & \cdots \\
1 & 1 & 1 & \cdots \\
1 & 1 & 1 & \cdots \\
\vdots & \vdots & \vdots & \ddots
\end{array}\right) .
$$

Or conversely, $B$ is diagonalized, and rescaled by $1 /(2 j+1)$, by acting with $V^{-1}$ and its transpose:

$$
P=V^{-1}[j] B V^{-1}[j]^{\text {transpose }} .
$$

Therefore, from $G=\left(V^{-1}\right)^{\dagger}\left(V^{-1}\right)$, the orthonormality relation may be written as a trace-projection of similarity transformed (i.e. conjugated) powers of $S$.

$$
\delta_{m, n}=\operatorname{Trace}\left[\left(V^{-1} S^{n} V\right) P\left(V^{-1} S^{m} V\right)^{\dagger}\right],
$$

where, in the chosen basis that diagonalizes $S$, there is no distinction between hermitian conjugation and taking the transpose since all matrices are real. However, having written it in this form, the relation (F5) is valid in any basis upon using suitably transformed $V$ and $P$. Note that $P$ will remain a projector in any other basis obtained by a similarity transformation. Also, $P$ will be hermitian and positive-semi-definite in any other basis obtained by a unitary transformation.

Compare the result in (F5) with the usual trace-norm of an arbitrary matrix $M$, namely, Trace $\left(M M^{\dagger}\right)$. As has been shown here, orthonormality of the powers of $V^{-1} S V$ results from a modified product within the trace, a modification produced simply by inserting $P$, albeit a singular matrix. As is well-known, associativity is not destroyed by such insertions to produce modified products of three or more matrices, hence it may be useful to think of the modification as a matrix star product, with $M_{1} \star M_{2} \equiv M_{1} P M_{2}$. The fact that $P$ is singular should not be cause for much concern, at least for the problem at hand, since already $S$ is not invertible for integer $j$.

As previously suggested, these trace-norm and modified product results are somewhat analogous to those for quantum distribution functions on phase space [16. A well-known result for discretely indexed purestate Wigner functions, $\mathrm{W}_{a}(x, p)$, is that $2 \pi \hbar \int \mathrm{W}_{a}(x, p) \star \mathrm{W}_{b}(x, p) d x d p=\delta_{a, b}$ where the star product is that discovered by Groenewold, namely, $\star=\exp \frac{i \hbar}{2}\left(\overleftarrow{\partial}_{x} \vec{\partial}_{p}-\overleftarrow{\partial}_{p} \vec{\partial}_{x}\right)$. In this case the Wigner functions are actually their own duals in the sense that the modified product may be eliminated by phase-space integrations by parts without changing the result (assuming there are no net contributions from the boundaries of phase space). Thus $2 \pi \hbar \int \mathrm{W}_{a}(x, p) \mathrm{W}_{b}(x, p) d x d p=\delta_{a, b}$. Moreover, in this case the modified product itself reduces to $2 \pi \hbar \mathrm{W}_{a}(x, p) \star \mathrm{W}_{b}(x, p)=\mathrm{W}_{a}(x, p) \delta_{a, b}$ with the individual Wigner functions normalized to $\int \mathrm{W}_{a}(x, p) d x d p=$ 1. But none of these last three statements apply by analogy to powers of spin matrices, their modified products, or their traces, at least not as described above.

Other analogies can be found for other phase space distributions [17. For example, Husimi distributions, $\mathrm{H}_{a}(x, p)$, also obey $2 \pi \hbar \int \mathrm{H}_{a}(x, p) * \mathrm{H}_{b}(x, p) d x d p=\delta_{a, b}$ with an appropriately modified $*$ product, but in this case the functions are not their own duals because integrations by parts do not eliminate the $*$. Rather, the dual functions are given by Glauber-Sudarshan distributions, $\mathrm{GS}_{a}$, such that $2 \pi \hbar \int \mathrm{GS}_{a}(x, p) \mathrm{H}_{b}(x, p) d x d p=\delta_{a, b}$, a result that is more closely analogous to the matrix statement (19). 


\section{Appendix G: Periodicized monomial approximations}

The coefficients $A_{0,1,2,3,4,5}^{[j]}(\theta)$ are plotted here for $j=69$ in blue, and for $j=137 / 2$ in red. The slope of each red (fermionic) curve plotted on the right is given exactly by the red curve plotted to its immediate left. Similarly, the slope of any blue (bosonic) curve on the left is given by the blue curve to its right, but in the row above.
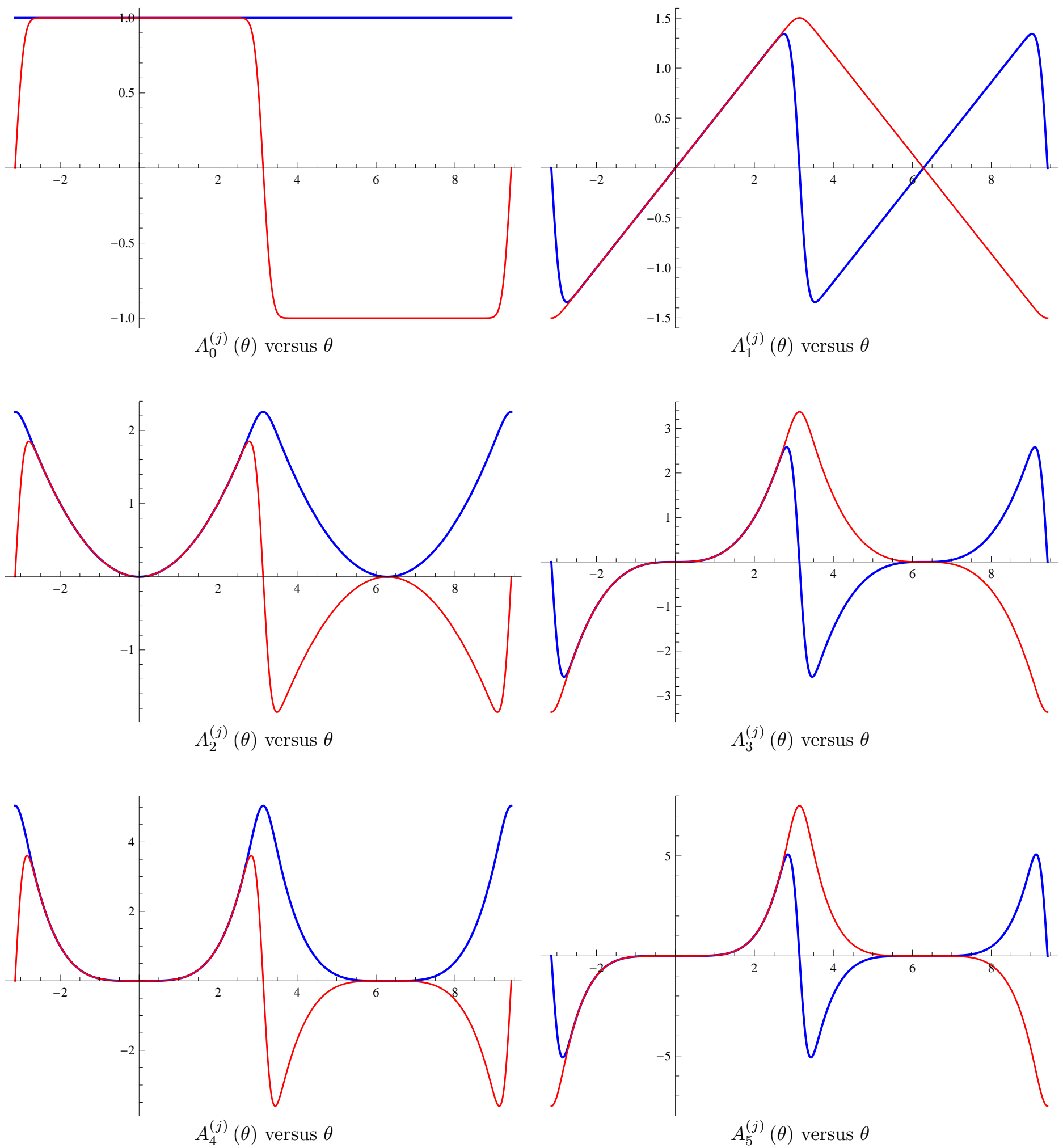